\title{
ECHOCARDIOGRAPHIC ASSESSMENT OF RIGHT VENTRICULAR MYOCARDIAL INFARCTION
}

\author{
Raghothaman Sethumadhavan ${ }^{1}$, Sureshkumar Ponnusamy²
}

${ }_{1}^{1}$ Senior Assistant Professor, Department of Cardiology, Chengalpattu Medical College, Kancheepuram, Tamilnadu, India. ${ }^{2}$ Assistant Professor, Department of Cardiology, Chengalpattu Medical College, Kancheepuram, Tamilnadu, India.

\begin{abstract}
BACKGROUND
Inferior wall myocardial infarction is complicated by right ventricular infarction in as much as 50\% of cases. In patients with RVMI, risk of major complications ${ }^{1}$ and death in the hospital are greater. Establishing the presence of RVMI in living patients is difficult because of right ventricular dysfunction and frequently it is transient in nature. Echocardiography ${ }^{3}$ provides a readily accessible tool for the evaluation of right ventricular function.

Aims and Objectives-To compare various echo methodologies in assessing right ventricular function in the setting of inferior wall myocardial infarction and its prognostic significance in assessing risk of mortality in various sub groups.

Setting and Design- This study was performed in the Department of Cardiology, Chengalpattu Medical College Hospital. 324 consecutive patients admitted with IWMI during the period of March 2016 - February 2018, were included in the study. The study is a prospective observational study.
\end{abstract} ABSTRACT

\section{MATERIALS AND METHODS}

Group 1 - Patients with IWMI with RVMI on ECG $(\mathrm{n}=151)$

Group 2 - Patients with IWMI without RVMI on ECG $(\mathrm{n}=173)$

Transthoracic echocardiography was performed in all patients within 48 hours of symptom onset, within 24 hours whenever feasible. Comparison of measurements between Group 1 and group 2 was performed using a two tailed Student's t-test.

\section{RESULTS}

- $\quad$ RV end-diastolic diameter was increased in patients with RVMI. Tricuspid regurgitation was noted in half of the patients with right ventricular involvement. It was mild in majority of cases.

- $\quad$ The TAPSE was statistically significantly decreased in patients with right ventricular myocardial infarction. MPI is raised to nearly two-fold the reference values in patients with RVMI.

\section{CONCLUSION}

Right ventricular dimension \& contractility were insignificant in detecting RVMI. Right ventricular systolic velocity in patients with RVMI was less compared to patients without RVMI. Increased MPI values were associated with higher mortality. RV dysfunction was worse in those whose echo was done within $24 \mathrm{hrs}$.

\section{KEY WORDS}

RVMI- Right Ventricular Myocardial Infarction, MPI - Myocardial Performance Index, TDI - Tissue Doppler Imaging, TAPSE Tricuspid Annular Peak Systolic Excursion, Sm - Myocardial systolic velocity, Em - Early phase of diastolic myocardial velocity, Am - Late phase of diastolic myocardial velocity.

HOW TO CITE THIS ARTICLE: Sethumadhavan R, Ponnusamy S. Echocardiographic assessment of right ventricular myocardial infarction. J. Evolution Med. Dent. Sci. 2018;7(47):5063-5068, DOI: 10.14260/jemds/2018/1126

\section{BACKGROUND}

Inferior wall myocardial infarction is complicated by right ventricular infarction (RVMI) in as much as $50 \%$ of cases.

In patients with RVMI, the risk of death in the hospital is high and incidence of major complications ${ }^{1}$ is greater. Right ventricular infarction contributes markedly to hemodynamic instability, atrio-ventricular conduction blocks ${ }^{3}$, cardiogenic shock $^{4}$, pulmonary hypertension and in-hospital mortality ${ }^{5}$ in patients with inferior wall myocardial infarction.

'Financial or Other Competing Interest': None.

Submission 09-10-2018, Peer Review 01-11-2018,

Acceptance 09-11-2018, Published 19-11-2018.

Corresponding Author:

Sureshkumar Ponnusamy,

Assistant Professor, Department of Cardiology,

Chengalpattu Medical College,

Kancheepuram, Tamilnadu, India.

E-mail: psureshkumar79@gmail.com

DOI: $10.14260 /$ jemds/2018/1126
Establishing the presence of RVMI in living patients is difficult because right ventricular dysfunction and frequently it is transient in nature. Criteria have been set for diagnosis of RVMI; but, even when strictly employed, the criteria lead to underestimation of the true incidence of right ventricular infarction.

There are a lot of problems in assessing the function of the right ventricle because-

a. Right ventricle is crescentic and truncated into separate inflow and outflow portions.

b. Endocardial boundary is not accurately localizable due to variable trabeculation pattern.

c. Right ventricle has a complex wall motion with the inflow portion contracting before the trabecular and outflow portion.

d. Right ventricle is located behind the sternum and hence is inaccessible to imaging.

e. There is an inter-dependence between right and left ventricle and this influences the RV ejection ${ }^{6}$ 


\section{Diagnosis of RVMI by Electrocardiography ${ }^{7}$}

ST elevation in V4R $>1 \mathrm{~mm}$ is the most commonly used criteria. This is $88 \%$ sensitive and $78 \%$ specific. ${ }^{8}$ The problems with using right sided chest leads for the diagnosis for right ventricular myocardial infarction is that ST elevation can be present in V4R in 18\% of patients even in the absence of RVMI, especially in pericardial disease \& acute pulmonary embolism. Also, this finding of ST elevation in right sided leads disappears after 10 hours in at least $50 \%$ of cases.

Various non-invasive assessment tools for right ventricular function:
a. Echocardiography 9
b. Radionuclide angiography:
c. Cardiac computed tomography:
d. Cardiac magnetic resonance:
e. Invasive assessment of right ventricular function:

Each of these has its own limitations and is expensive and availability is limited. So, echocardiography ${ }^{2}$ provides a readily accessible tool for the evaluation of right ventricular function.

\section{Aim of the Study}

- To compare various echo methodologies in assessing Right Ventricular function in the setting of Inferior wall myocardial infarction.

- To assess the echocardiographic and clinical parameters associated with increased mortality.

- To assess occult RV dysfunction by echocardiography in patients without RVMI on ECG.

- To compare RV function in patients who are thrombolysed \& those not thrombolysed.

- To assess RV function by echocardiography done at different time intervals after the onset of myocardial infarction.

- To compare RV function in males \& females and in different age groups.

\section{MATERIALS AND METHODS}

This study was performed in the Department of Cardiology, Chengalpattu Medical College Hospital, for 324 consecutive patients admitted with IWMI during the period of March 2016 - February 2018, for a period of 2 years. The study is a prospective observational study involving 324 patients.

\section{Study Group Selection}

Ethical committee clearance was obtained to conduct the study in our hospital. All subjects provided written informed consent to participate in the study before inclusion.

\section{Inclusion Criteria}

1. IWMI with and without RVMI on ECG.

2. First MI (MI diagnosed by history, ECG \& enzymes).

3. Within 48 hours of onset of chest pain.

4. Any age group.

5. Both sexes

6. Both thrombolysed \& not thrombolysed patients.

\section{Exclusion Criteria}

1. COPD/PPH/Cor pulmonale

2. Valvular HD/Congenital HD.
3. Previous MI.

4. Complete heart block, Arrhythmias during echo.

5. DCM/LHF of any cause

6. Technically inadequate echo.

\section{Patient Characteristics}

The study population included 324 patients (178 male and 146 female) admitted to the Intensive Care Unit of Chengalpattu Medical College Hospital, Chengalpattu - 01, with a first $Q$ wave acute inferior myocardial infarction with or without RVMI.

\section{The Patients were divided into Two Groups-}

1. Group 1 - Patients with IWMI with RVMI $(n=151)$

2. Group 2 - Patients with IWMI without RVMI $(\mathrm{n}=173)$

Out of the 324 patients, 32 patients (14 from Group 1 \& 18 from Group 2) were excluded from the study due to various reasons described below-

- Delayed presentation $>2$ days after onset of chest pain $(n=14)$

- Presence of preexisting cardiac dysfunction due to myocardial infarction, cor-pulmonale, DCM or valvular heart disease $(n=8)$

- Atrial fibrillation $(n=3)$

- Technically inadequate echo $(n=7)$.

Finally, Group 1 (IWMI with RVMI) consisted of 137 patients and Group 2 (IWMI without RVMI) consisted of 155 patients, and these 292 patients underwent clinical evaluation, investigations including EKG \& Echocardiography.

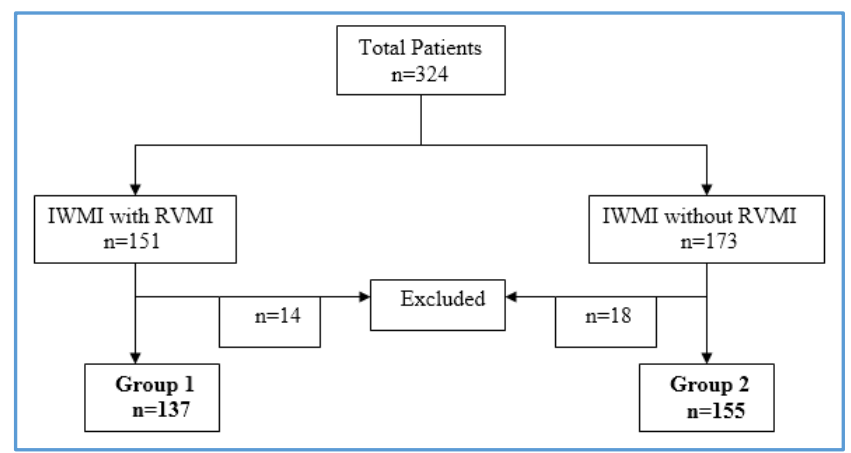

Detailed history was obtained from all the patients, Baseline investigations were done in all patients. Cardiac enzymes, namely, Troponin $\mathrm{T}$ and CK-MB were done in all patients.

\section{Patient Characteristics}

Patient among the two groups were almost equally distributed age \& sex wise, and presence and absence of risk factors. Their hemodynamic profile was also almost matching and the percentage of patients who were thrombolysed were also almost equal ( $68 \%$ vs $64 \%$ )

Patients who were in the time window for thrombolysis, and who had no absolute contra-indications for thrombolysis were given Inj. Streptokinase 10 I.V over 45 minutes via an infusion. All patients were treated with antiplatelets, statins, and intravenous fluid replacements whenever needed. Those who developed significant atrioventricular blocks were managed with temporary pacemaker implantation. Those in shock were managed with fluid replacement and inotropic 
support. All patients were closely monitored during the intensive period.

\section{Echocardiography}

Transthoracic echocardiography 11 was performed in all patients within 48 hours of symptom onset, within 24 hours whenever feasible using an Esaote Mylab 40 echocardiography machine with a $3.5 \mathrm{MHz}$ transducer including second harmonic and tissue Doppler imaging technology.

The following Measurements were done-

1. RV dimensions.

2. RV contractile function \& Inter-ventricular septum movement.

3. Tricuspid regurgitation jet \& Pulmonary artery systolic pressure. 12

4. Tricuspid annular movement - M-mode.

5. Doppler tissue imaging - lateral \& septal wall systolic/ early, late diastolic velocities.

6. Myocardial performance index (Tei index).

\section{RV dimensions}

It was done using the same M-mode cross-section in the parasternal long axis view. Normal values were defined as less than $26 \mathrm{~mm}$ for the right ventricular end diastolic diameter, greater than $37 \mathrm{~mm}$ for the left ventricular end diastolic diameter, and less than 0.5 for the ratio of the right ventricular diameter to the left ventricular diameter. ${ }^{5}$

\section{Right Ventricular Contractile Function}

Right ventricular wall motion was assessed in parasternal long axis ${ }^{13}$, parasternal short axis, RV inflow and apical four chamber views. Each segment was analysed as normal, hypokinetic, akinetic and dyskinetic.

\section{Tricuspid Regurgitation Jet}

Tricuspid regurgitant flow peak velocity was recorded from apical four chamber view using colour doppler ${ }^{14}$ and scored as 0 -none, 1-mild, 2-moderate and 3-severe TR. The systolic tricuspid regurgitation pressure gradient between the RV and the right atrium was calculated by the simplified Bernoulli equation ${ }^{15,} 16$

\section{Tissue Doppler Index}

Pulsed wave TDI ${ }^{17}$ of the systolic tricuspid annular motion ${ }^{13}$, $18(\mathrm{~cm} / \mathrm{s})$ at the lateral free wall (TVlat) and at the septal wall (TVsept) was obtained from the apical 4-chamber view using a pulsed wave doppler sampling gate of 2-4 $\mathrm{mm}$ and a sweep of $100-150 \mathrm{~mm} / \mathrm{s}$, as follows: one during the early phase of diastolic myocardial velocity (Em) and another during the late phase of diastolic myocardial velocity (Am) ${ }^{19,} 20$.

\section{Myocardial Performance Index 21}

Right ventricular myocardial performance was calculated as follows. The tricuspid inflow is interrogated by pulse wave doppler in the apical 4 chamber view and the time interval from end of ' $A$ ' wave to the onset of the following ' $E$ ' wave is noted as ' $a$ ' in milliseconds. Then the pulmonary valve is interrogated by pulse wave doppler in the parasternal short axis view. The total pulmonary ejection time is noted as ' $b$ '. 5 consecutive cardiac cycles were averaged to obtain each value for ' $a$ ' and 'b' to correct for heart rate variation and measurement errors.

The time interval ' $a$ ' denotes combination of isovolumetric contraction time (IVCT), ${ }^{22}$ ejection time (ET) and isovolumetric relaxation time (IVRT). As the doppler period ' $b$ ' is the pulmonary ET, the sum of IVCT and IVRT is derived by subtracting ' $b$ ' from ' $a$ '. MPI23,24 is calculated as MPI $=\mathrm{a}-\mathrm{b} / \mathrm{b}$ and $\mathrm{a}$ valueless ratio is obtained.

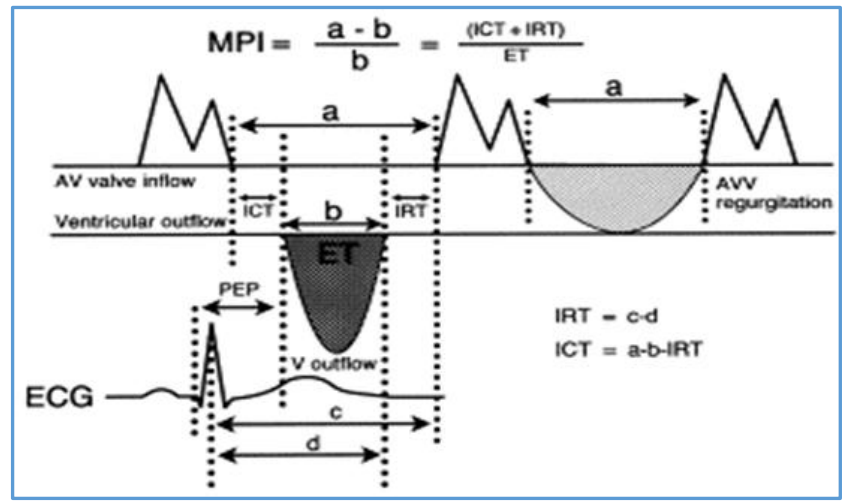

\section{Statistical Analysis}

Data are presented as Mean \pm SD for descriptive statistics. We chose the average values for 3-time measurements. Continuous data are expressed as mean SD. Comparison of measurements between Group 1 and group 2 was performed using a two tailed Student's t-test. A probability (P) value of less than 0.05 was considered significant.

\section{RESULTS}

Most of the patients were in the age group of 40-60 years (75\%). Most of the study population were male patients (78\%). 38\% were hypertensives, $46 \%$ had a history of diabetes mellitus, $58 \%$ of patients were smokers, and a family history of ischemic heart disease was evaluated in $23 \%$.

Various Complications were noted in the Study Patients, the Commonest being Conduction Disturbances

- Conduction disturbances were noted in $34 \%$ of Group 1 , and in only $10 \%$ of patients in Group 2.

- Cardiogenic shock complicated 7\% of Group 1, and none of the patients in Group 2.

- Pulmonary artery systolic pressures $>30 \mathrm{mmHg}$ was noted in $69 \%$ of Group 1, while in Group 2 patients, only $4 \%$ had PASP exceeding $30 \mathrm{mmHg}$.

28 patients died due to complications - of which 21 patients belonged to Group 1 and 7 patients belonging to Group 2 died. All patients died within 1 week of the acute event.

\section{Electrocardiography}

Evidence of right ventricular involvement as defined by $1 \mathrm{~mm}$ ST elevation in V4R was fund in 210 patients (of which, 164 patients had proximal RCA as the culprit vessel on ECG and 46 had left circumflex as the vessel causing myocardial infarction).

\section{Echocardiography}

Echocardiography was done in all 324 patients within 48 hours after the onset of the acute event, of which, 
echocardiography was done within 24 hours of symptom onset in 198 patients. The results of the investigation is as below-

- $\quad$ RV end-diastolic diameter was increased in patients with RVMI, compared with those without RV involvement, although it failed to achieve statistical significance $(\mathrm{p}=0.1)$.

- RV contraction abnormalities and IV septal motion abnormalities were noted in $41 \%$ of patients with RVMI, and only in $3 \%$ of patients without it. Significantly, the wall motion abnormalities were noted in those patients who had their echo done within 24 hours of symptom onset, stressing the fact that, the right ventricle recovers fast after an ischemic insult.

- Tricuspid regurgitation was noted in nearly half of the patients with right ventricular involvement; this regurgitation, when quantified by colour doppler, was mild in a majority of cases, moderate in some and severe in none.

- The pulmonary artery systolic pressure was slightly elevated and above normal in patients of Group 1, compared to Group 2 (Picture 10), statistically not significant $(\mathrm{p}=0.2)$

- The tricuspid annular excursion was statistically significantly decreased in patients with right ventricular myocardial infarction, signifying that $\mathrm{RV}$ systolic function was depressed in those patients $(\mathrm{p}=0.05)$.

- Myocardial performance index was found to be statistically significant, it is raised to nearly two-fold the reference values in patients with RVMI; the values were not much high compared to controls in those without right ventricular involvement $(\mathrm{p}=0.01)$.

- $\quad$ Tissue doppler - It was found that the right ventricular free wall $\mathrm{Sm}$ and $\mathrm{Em}$ values were statistically significantly depressed in patients with right ventricular myocardial infarction $(\mathrm{p}=0.02)$. The values were near normal in those without RV involvement.

\begin{tabular}{|c|c|c|}
\hline Parameters & Group 1 (n=44) & Group 2 (n=60) \\
\hline RV EDD (mm) & $21.8 \pm 4.1$ & $11.3 \pm 3$ \\
\hline $\begin{array}{c}\text { RV contraction } \\
\text { abnormalities }\end{array}$ & $(41 \%)$ & $(3 \%)$ \\
\hline $\begin{array}{c}\text { IVS motion } \\
\text { abnormality }\end{array}$ & $(31 \%)$ & $(2 \%)$ \\
\hline $\begin{array}{c}\text { Tricuspid } \\
\text { regurgitation }\end{array}$ & $(48 \%)$ & $(10 \%)$ \\
\hline PASP (mmHg) & $31.8 \pm 2.7$ & $17.1 \pm 2.8$ \\
\hline TAPSE (mm) & $14.1 \pm 1.2$ & $18.2 \pm 0.71$ \\
\hline MPI & $0.44 \pm 0.07$ & $0.28 \pm 0.02$ \\
\hline \multirow{2}{*}{\begin{tabular}{c} 
TDI - RV Free wall Sm \\
(cm/sec) Em \\
\cline { 2 - 3 } Am
\end{tabular}} & $9.45 \pm 0.49$ & $12.1 \pm 0.52$ \\
\cline { 2 - 3 } & $6.8 \pm 0.34$ & $8.3 \pm 0.65$ \\
\hline $\begin{array}{c}\text { TDI - Septal wall } \\
\text { (cm/sec) Sm }\end{array}$ & $6.3 \pm 0.5$ & $9.4 \pm 0.65$ \\
\hline
\end{tabular}

Table 1. Values of various echocardiographic parameters in patients presenting with IWMI with or without RVMI

\section{Sub Group Analysis}

- All echocardiographic parameters did not statistically significantly differ in the various age groups $(\mathrm{p}=0.5)$.

- Similarly, the parameters also did not statistically significantly differ in both sexes, revealing that RV dysfunction was present equally in both males and females.

- As far as thrombolysis is concerned, RVEDD was higher, TAPSE was lower, $\mathrm{Sm} \& \mathrm{Em}$ values were lower in patients thrombolysed when compared to those who were not; but, they were statistically insignificant $(\mathrm{p}=0.2)$. Only, myocardial performance indeed was statistically significantly low $(p=0.04)$ in patients who were thrombolysed with streptokinase when compared to those who were not.

- Echocardiography done within 24 hours of the acute event, showed significantly lower Sm scores and very significant high MPI scores in patients with right ventricular myocardial infarction.

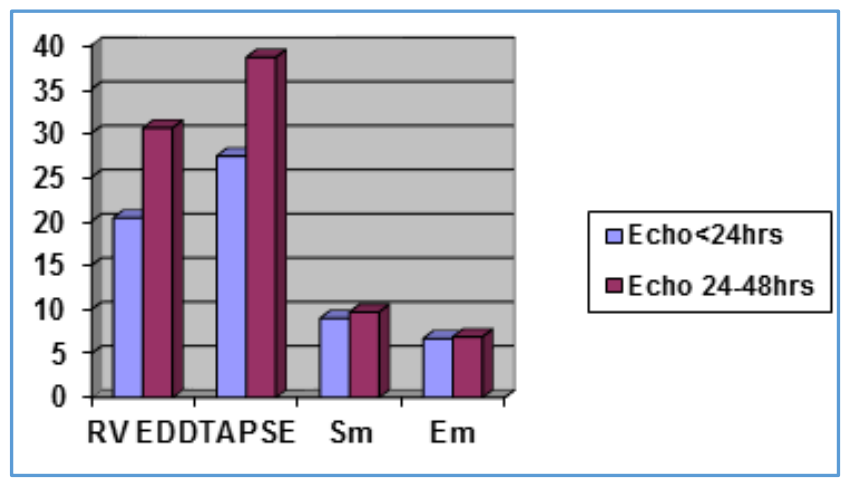

\section{ECG \& Echo Correlation}

- Echo identified RVMI in 9 patients who did not show RVMI on ECG.

- 11 patients with RVMI ECG did not have evidence of RVMI on echo.

\section{DISCUSSION}

In patients with RVMI, the risk of death in the hospital is high and major complications are greater.

\section{Clinical Implications of RVMI}

The severity of the hemodynamic abnormalities associated with RV infarction is related to the extent of RV ischemia and consequent RV dysfunction as well as to the restraining effect of the pericardium, LV function, and ventricular interdependence. It has now been shown both experimentally and clinically that the intact LV may assist RV ejection by LV septal contraction causing a bulging into the RV which generates an active RV systolic pressure wave and systolic force sufficient for pulmonary perfusion. Loss of this mechanism with concomitant LV infarction, particularly when the interventricular septum is involved, may lead to further hemodynamic deterioration in patients with RV infarction. Furthermore, augmented atrial contraction is necessary to overcome the stiffness of the ischemic RV, and factors that impair RV filling (intravascular volume depletion, concomitant atrial infarction, and loss of atrioventricular synchrony) may severely compromise haemodynamics and result in cardiogenic shock.

RVMI recovers fast and suffers less ischemic insult because:

- The right ventricle has lesser muscle mass and thickness, hence the infarct is not huge 
- The coronary perfusion through the Right Coronary artery occurs in both systoly and diastoly.

- There is direct diffusion of oxygen from the right ventricular cavity.

\section{Newer Diagnostic Modalities}

- Contrast echocardiography: 25

- Three-Dimensional Echocardiography: 26

- Automated border detection methods:

- Intra-cardiac echocardiography:

- Strain \& strain rate imaging:

\section{Comparison with Other Studies:}

Right Ventricular Contraction Abnormalities

In the same study by Dokainish et al, right ventricular contraction abnormalities were present in $11 / 22$ patients $(60 \%)$ with right ventricular myocardial infarction and only in 6/28 (20\%) patients without right ventricular involvement and it had statistical significance with $\mathrm{p}=<0.02$.

In our study, RV contraction abnormalities and IV septal motion abnormalities were noted in $41 \%$ of patients with RVMI, and only in $3 \%$ of patients without it.

\section{Tricuspid Regurgitation Jet}

Anna Vittoria et al found that tricuspid regurgitation was detected in 26 out of 44 Patients with right ventricular myocardial infarction. The mean peak velocity of tricuspid regurgitation was $3.8 \pm 0.8 \mathrm{~m} / \mathrm{s}$.

In our study, tricuspid regurgitation was noted in $48 \%$ patients with right ventricular involvement; this regurgitation, when quantified by colour doppler, was mild in a majority of cases, moderate in some and severe in none.

\section{Tricuspid Annular Peak Systolic Excursion (TAPSE):}

In a study conducted by Alam et al, from the echocardiographic apical 4-chamber views, TAPSE was recorded at the RV free wall with the use of 2-dimensional guided M-mode recordings. The tricuspid annular motion 27 was reduced in inferior $\mathrm{Ml}$ compared with that in healthy individuals ( 20.5 and $25 \mathrm{~mm}, \mathrm{P}<.001$ ).

In our study, the tricuspid annular excursion was significantly decreased in patients with right ventricular myocardial infarction (14.1 $\pm 2.4 \mathrm{~mm}$ Vs. $18.2 \pm 1.9)$, signifying that RV systolic function was depressed in those patients

\section{Myocardial Performance Index (Tei Index)}

Tei and coworkers suggested an easily obtainable Dopplerderived index of right ventricular dysfunction, which combined elements of systolic and diastolic function. A RV MPI values of $>0.65$ is associated with an increased mortality. In our study, Myocardial performance index was found to be statistically significant, it was $0.45 \pm 0.07$ in patients with RVMI, compared to near normal values of $0.29 \pm 0.04$ in patients without right ventricular infarction.

\section{Tissue Doppler Imaging:}

In an extensive adult patient population, a Swiss study demonstrated that TDI of the systolic lateral tricuspid annular long axis velocity (TVlat) is accurate to characterize systolic RV function independent of most, pathophysiologically meaningful cofactors. A velocity of 12 and $9 \mathrm{~cm} / \mathrm{s}$ differentiates among normal and moderately reduced RV ejection fraction, respectively between moderately and severely impaired RV EF.

In our study, it was found that the right ventricular free wall $\mathrm{Sm}$ and $\mathrm{Em}$ values were statistically significantly depressed in patients with right ventricular myocardial infarction compared to those without RV involvement. ( $\mathrm{Sm}$ $9.4 \pm 0.7$ Vs. $12.1 \pm 1.1$; Em $6.8 \pm 0.7$ Vs. $8.4 \pm 1.0$ ).

\section{CONCLUSION}

- Age and sex did not significantly correlate with right ventricular function after a myocardial infarction.

- Right ventricular dimension \& contractility were insignificant in detecting RVMI.

- On tissue doppler, right ventricular free wall systolic velocity and early diastolic velocity in patients with RVMI was less compared to patients without RVMI and the correlation was statistically significant.

- Right ventricular myocardial performance index (Tei index) correlated with RV dysfunction and attained statistical significance. Increased MPI values were also associated with higher mortality.

- $\quad$ RV dysfunction was worse in those whose echo was done within $24 \mathrm{hrs}$, when compared with those done after showing that RV function improves with time.

- Echo can identify right ventricular dysfunction in patients who have no or doubtful findings of RVMI on ECG.

\section{ACKNOWLEDGEMENT}

We would like to acknowledge the support of the Medical College Dean, the Medical Superintendent and the administrative staff for giving us the necessary permissions to carry out the study.

Also, we wish to thank the support staff and paramedical staff of the Department of Cardiology for helping us with the echocardiography.

We wish to thank the patients who participated in the study.

\section{REFERENCES}

[1] Cohn JN, Guiha NH, Broder MI, et al. Right ventricular infarction: clinical and hemodynamic features. Am J Cardiol 1974;33(2):209-14.

[2] Levine RA, Gibson TC, Aretz T, et al. Echocardiographic measurement of right ventricular volume. Circulation 1984;69(3):497-505

[3] Marvic Z, Zaputovic L, Matana A, et al. Prognostic significance of complete atrioventricular block in patients with acute inferior myocardial infarction with and without right ventricular involvement. Am Heart J 1990;119(4):823-8.

[4] Jacobs AK, Leopold JA, Bates E, et al. Cardiogenic shock caused by right ventricular infarction: a report from the SHOCK registry. J Am Coll Cardiol 2003;41(8):1273-9.

[5] Garty I, Barzilay J, Bloch L, et al. The diagnosis and early complications of right ventricular infarction. Eur J Nucl Med 1984;9(10):453-60.

[6] Clyne CA, Alpert JS, Benotti JR. Interdependence of the left and right ventricles in health and disease. Am Heart J 1989;117(6):1366-73. 
[7] Erhardt LR, Sjogren A, Wahlberg I. Single right-sided precordial lead in the diagnosis of right ventricular involvement in inferior myocardial infarction. Am Heart J 1976;91(5):571-6.

[8] Bellamy GR, Rasmussen HH, Nasser FN, et al. Value of two-dimensional echocardiography, electrocardiography and clinical signs in detecting right ventricular infarction. Am Heart J 1986;112(2):304-9.

[9] Geva T, Powell AJ, Crawford EC, et al. Evaluation of regional differences in right ventricular systolic function by acoustic quantification echocardiography and cine magnetic resonance imaging. Circulation 1998;98(4):339-45.

[10] Berger PB, Ruocco NA, Timm CT, et al. The impact of thrombolytic therapy in right ventricular infarction complicating inferior myocardial infarction: results from TIMI II. Circulation 1989;80:313.

[11] Kaul S, Tei C, Hopkins JM, et al. Assessment of right ventricular function using two-dimensional echocardiography. Am Heart J 1984;107(3):526-31.

[12] Garcia-Fernandez MA, Cotrim C, Moreno M, et al. Prediction of right ventricular ejection fraction by Doppler determination of right systolic time intervals. Rev Port Cardiol 1995;14(6):447, 451-8.

[13] Meluzin J, Spinarova L, Bakala J, et al. Pulsed Doppler tissue imaging of the velocity of tricuspid annular systolic motion; a new, rapid and non-invasive method of evaluating right ventricular systolic function. Eur Heart J 2001;22(4):340-8.

[14] Tuller D, Steiner M, Wahl A, et al. Systolic right ventricular function assessment by pulsed wave tissue Doppler imaging of the tricuspid annulus. Swiss Med Wkly 2005;135(31-32):461-8.

[15] Dokainish H, Gin K, et al. Tissue Doppler echocardiography of the tricuspid annulus in the detection of right ventricular infarction in acute inferior wall myocardial infarction. Echocardiography 2005;23(4):287-94.

[16] Garcia-Fernandez MA, Lopez-Sendon J. Two dimensional echocardiography and Doppler in the right ventricular infarction. Rev Port Cardiol 1990;9(3):22744.
[17] Mattioli AV. Doppler echocardiographic evaluation of right ventricular function in patients with right ventricular infarction. J Ultrasound Med 2000;19:831-6. 0278-4297

[18] Alam M, Wardell J, Andersson E, et al. Right ventricular function in patients with first inferior myocardial infarction: assessment by tricuspid annular motion and tricuspid annular velocity. Division of Cardiology, Karolinska Institute at South Hospital, Sweden. Am Heart J 2000;139(4):710-5.

[19] Mattioli AV, Bastia E, Mattioli G. Doppler Echocardiographic findings in patients with right ventricular infarction. J Ultrasound Med 1998;17(5):297-301.

[20] Yilmaz M, Erol MK, Acikel M, et al. Pulsed Doppler tissue imaging can help to identify patients with right ventricular infarction. Heart \& Vessels 2003;18(3):1126.

[21] Tei C. New non-invasive index for combined systolic and diastolic ventricular function. J Cardiol 1995;26(2):135-6.

[22] Lindqvist $P$, Waldenstrom A, Wikstrom G, et al. The use of isovolumic contraction velocity to determine right ventricular state of contractility and filling pressures: a pulsed Doppler tissue imaging study. Euro J Echocardiography 2005;6(4):264-70.

[23] Ozdemir K, Altunkeser BB, Lcli A, et al. New parameters in identification of right ventricular myocardial infarction and proximal right coronary artery lesion. Chest 2003;124(1):219-26.

[24] Alagesan R, Chockalingam A. Myocardial performance index in the diagnosis of right ventricular myocardial infarction. Echocardiography 10/2004;21(7):639-43.

[25] Tokgozoglu SL, Caner B, Kabakci G, et al. Measurement of right ventricular ejection fraction by contrast echocardiography. Int J Cardiol 1997;59(1):71-4.

[26] Wang XF, Li ZA, Cheng TO, et al. Clinical application of three-dimensional transesophageal echocardiography. Am Heart J 1994;128(2):380-8.

[27] Hammarstrom E, Wranne B, Pinto FJ, et al. Tricuspid annular motion. J Am Soc Echocardiogr 1991;4(2):1319. 